\title{
Salud bucal y factores relacionados en pacientes portadores de prótesis bimaxilar en una institución docencia- servicio de Medellín, Colombia
}

\author{
Miguel F. Gómez-Pino, Od, María Alejandra Silva-Ríos, Est Odon ${ }_{1}$, \\ María Camila Páez-Herrera, Est Odon 1 , Juliana Gómez-Ortega, Od, \\ Francisco J. Zea-Restrepo, Esp ${ }_{1}$, Andrés A. Agudelo-Suárez, Ph.D.
}

, Facultad de Odontología, Universidad de Antioquia, Medellín, Colombia

Recibido: 23 de junio del 2016 Aprobado: 15 de agosto del 2016

*Autor de correspondencia: Miguel F. Gómez-Pino. Facultad de Odontología, Universidad de Antioquia, Medellín, Colombia. Calle 70 n.5 52-21. Teléfono: (57) 421967 72. Correo electrónico: gomezpinom@gmail.com

Cómo citar este artículo: Gómez-Pino MF, Silva-Ríos MA, Páez-Herrera MC, Gómez-Ortega J, Zea-Restrepo FJ, AgudeloSuárez AA. Salud bucal y factores relacionados en pacientes portadores de prótesis bimaxilar en una institución docenciaservicio de Medellín, Colombia. Rev Nac Odontol. 2017;13(24):65-77. doi: http://dx.doi.org/10.16925/od.v12i24.1662

Resumen. Introducción: este estudio tuvo como objetivo analizar el estado de salud bucal y los factores relacionados en pacientes edentados portadores de prótesis total bimaxilar que acudieron a la Facultad de Odontología de la Universidad de Antioquia. Métodos: estudio descriptivo en 111 pacientes adultos atendidos en el período 20092016. Se realizó un examen clínico y una encuesta que recopiló información sociodemográfica, antecedentes personales, condiciones de higiene bucal, evaluación clínica de la ATM, estado, uso y satisfacción de las prótesis dentales, y, finalmente, evaluación de la calidad de vida relacionada con la salud bucal (CVRSB). Se realizó análisis de frecuencias absolutas y relativas según sexo para cada una de las variables consideradas. Resultados: la mayor parte de la población evaluada $(98,2 \%)$ vivía en estratos bajo y medio, y el 82,9\% de los pacientes cursaron estudios básicos. Más de la mitad de la población $(69,4 \%)$ reportó tener un estado de salud bueno, sin embargo, se encontró que el 53,2\% relató sufrir hipertensión y el 23,4\% diabetes. Tres cuartas partes de los pacientes evaluados manifestaron limpiarse la mucosa alveolar, tanto superior, como inferior (mayor en mujeres, 83,8\%). Las características protésicas más frecuentes en ambas prótesis en orden decreciente fueron pigmentaciones, cálculos y porosidades. La población encuestada expresó mayor satisfacción con la prótesis superior respecto de la inferior. Más de tres cuartas partes de la población estudiada tienen una baja CVRSB. Conclusiones: se requieren estrategias que contribuyan al mejoramiento de la calidad de la atención en salud bucal, reflejadas en actividades de seguimiento de los tratamientos efectuados en este segmento de la población.

Palabras clave: boca edentada, higiene bucal, prótesis dental total, salud bucal, servicios de integración docente asistencial. 


\title{
Oral health and related factors in patients with bimaxillary prosthesis at a teaching- service institution in Medellín, Colombia
}

\begin{abstract}
Introduction: This study aimed to analyze the state of oral health and related factors in edentulous patients with bimaxillary total prosthesis who attended the School of Dentistry at the Universidad de Antioquia. Methods: Descriptive study in 111 adult patients attended to in the period 2009-2016. A clinical examination and a survey, which compiled sociodemographic information, personal history, oral hygiene conditions, clinical evaluation of TMJ, state, use of and satisfaction with dental prostheses, and, finally, evaluation of oral health-related quality of life (OHRQOL), were carried out. Absolute and relative frequency analyses were performed according to sex for each of the variables considered. Results: Most of the population evaluated (98.2\%) lived in low and medium socio-economic levels, and $82.9 \%$ of the patients only went to elementary school. More than half of the population (69.4\%) reported having a good health status; however, it was found that $53.2 \%$ reported suffering from hypertension and $23.4 \%$ diabetes. Three-quarters of the patients evaluated stated that they clean their alveolar mucosa, both upper and lower (greater in women, 83.8\%). The most frequent prosthetic characteristics in both prostheses in decreasing order were pigmentations, calculus and porosities. The population surveyed expressed greater satisfaction with the upper prosthesis versus the lower one. More than three quarters of the population studied have a low OHRQoL. Conclusions: Strategies are needed to contribute to the improvement of the quality of oral healthcare, reflected in follow-up activities of the treatments provided to this segment of the population.
\end{abstract}

Keywords: edentulous mouth, oral hygiene, total dental prosthesis, oral health, assistance teaching integration services.

\section{Saúde bucal e fatores relacionados em pacientes portadores de prótese bimaxilar em uma instituição docência-serviço de Medellín, Colômbia}

Resumo. Introdução: este estudo teve como objetivo analisar o estado de saúde bucal e os fatores relacionados em pacientes edentados portadores de prótese total bimaxilar que recorreram à Faculdade de Odontologia da Universidade de Antioquia. Métodos: estudo descritivo com 111 pacientes adultos atendidos no período 2009-2016. Realizou-se um exame clínico e uma pesquisa que reuniu informação sociodemográfica, antecedentes pessoais, condições de higiene bucal, avaliação clínica da ATM, estado, uso e satisfação com as próteses dentárias, e, finalmente, avaliação da qualidade de vida relacionada com a saúde bucal (QVRSB). Foi realizada análise de frequências absolutas e relativas de acordo com o sexo para cada uma das variáveis consideradas. Resultados: a maior parte da população avaliada (98,2\%) vivia em estratos sociais baixo e médio e $82,9 \%$ dos pacientes cursaram estudos básicos. Mais da metade da população $(69,4 \%)$ reportou ter um bom estado de saúde, no entanto, descobriu-se que 53,2\% relatou sofrer de hipertensão e $23,4 \%$ de diabetes. Três quartos dos pacientes avaliados manifestaram que limpavam a mucosa alveolar, tanto superior quanto inferior (maior em mulheres, 83,8\%). As características protéticas mais frequentes em ambas as próteses em ordem decrescente foram pigmentações, cálculos e porosidades. A população entrevistada expressou maior satisfação com a prótese superior em relação à inferior. Mais de três quartos da população estudada têm uma baixa QVRSB. Conclusões: são necessárias estratégias que contribuam para o melhoramento da qualidade do atendimento em saúde bucal, refletidas em atividades de acompanhamento dos tratamentos efetuados nesse segmento da população.

Palavras-chave: boca edentada, higiene bucal, prótese dental total, saúde bucal, serviços de integração docente assistencial. 


\section{Introducción}

el edentulismo total o la ausencia total de dientes, es una condición que impacta en la calidad de vida de las personas que la presentan [1]. Se encuentra con mayor frecuencia en pacientes adultos y adultos mayores [2]. Como causas principales, se le atribuyen enfermedades tales como la caries dental y la enfermedad periodontal [3, 4], las cuales se consideran problemas de salud pública por su magnitud y severidad $[5,6]$. La pérdida de piezas dentales conlleva problemas estéticos, faciales y funcionales en la cavidad bucal [7], signos y síntomas relacionados a desordenes de la articulación temporomandibular [8], dificultades de fonación, trastornos nutricionales [9] y problemas cardiovasculares [10].

En Colombia, según datos del IV Estudio Nacional de Salud Bucal, el edentulismo total bimaxilar presenta una prevalencia del 7,4\% en la población de 45 a 64 años, y un 33\% para el grupo de 65 a 79 años de edad [11]. A nivel internacional, la prevalencia es variable entre países. En Brasil, por ejemplo, para el caso de los adultos de 65 y más años, la prevalencia fue del 54\% [12]. En Canadá, es del 6\%, incluyendo el grupo de 20 a 79 años, y del $22 \%$ en el grupo de 60 a 79 . En Turquía, es del $48 \%$ para el grupo de 65 a 74 años y, en México, del $26 \%$ para ese mismo grupo [13]. Cabe recalcar que en la pérdida dental intervienen factores individuales, patologías orales y otras de carácter sistémico [13], así como determinantes sociales y culturales $[7,14,15]$.

En cuanto a las necesidades en salud bucal, esta población requiere rehabilitación por medio de la prótesis total, la cual ha sido utilizada para devolver forma, función y estética al paciente edentado [16], así como a fin de mejorar su calidad de vida [17]. No obstante, se presentan dificultades relacionadas con el mantenimiento de las prótesis totales que afectan el éxito del tratamiento a mediano y largo plazo [18-20].

La Facultad de Odontología de la Universidad de Antioquia, como institución formadora de recurso humano en salud bucal, atiende bajo el modelo docencia-servicio una buena cantidad de adultos que requieren prótesis bimaxilares. Un estudio piloto realizado en la institución evidenció algunas dificultades en el estado y el mantenimiento de las prótesis, a la vez que sugirió la realización de nuevos estudios incorporando nuevas variables asociadas a la situación de salud bucal en estos pacientes [20]. Es importante estudiar la relación que existe entre el estado de salud bucal y la presencia de condiciones sistémicas, los hábitos de higiene específicos para este tipo de pacientes [19], así como los hábitos nocivos como el consumo de tabaco [21] y alcohol, y un análisis integral del sistema estomatognático [22]. De igual manera, cobra importancia reconocer las percepciones de los pacientes con referencia a la calidad de vida y su relación con la salud bucal [23], ya que se considera un importante predictor a nivel individual y colectivo, en razón a que se centra en las necesidades y las percepciones sobre la situación de las personas en aspectos relacionados con su actividades cotidianas con respecto a la salud bucal — bien sean físicas, psicológicas o sociales- $[17,23]$.

De acuerdo con lo anterior, el objetivo de este estudio es analizar el estado de salud bucal y sus factores relacionados en los pacientes edentados portadores de prótesis total bimaxilar, quienes acudieron a esta institución docencia-servicio de Medellín.

\section{Métodos}

Se trata de un estudio descriptivo. La población de estudio la constituyen los pacientes adultos atendidos en las clínicas de la Facultad de Odontología, a quienes previamente se les había instalado prótesis dental total bimaxilar. Se seleccionó una muestra por conveniencia, y como criterios de inclusión se consideraron aquellos pacientes que habían acudido a la Facultad de Odontología de la Universidad de Antioquia en el período 2009-2015, cuyas prótesis fuesen fabricadas e instaladas dentro de la facultad, pacientes mayores de 49 años de edad y que voluntariamente aceptaran participar. Se excluyeron aquellos que portaran prótesis no convencionales tales como las sobredentaduras o implantosoportadas, así como a aquellos pacientes cuyo compromiso sistémico les impidiera asistir a la revisión en la institución.

Para la selección de la población, se utilizaron los criterios establecidos en un estudio piloto anterior, realizado por dos de los coautores del presente artículo [20]. A partir de la base de datos suministrada por la institución, se depuraron los pacientes que hubiesen pagado "Impresión definitiva con Pasta Zinquenólica", y se seleccionaron 990 
historias, de las cuales se revisaron 802. Al depurar la información, se encontró que 373 pacientes cumplían con los criterios de inclusión, pero la muestra definitiva se realizó con 111 pacientes que aceptaron participar. En la figura 1 se muestra el proceso de selección de los pacientes incluidos definitivamente en el estudio.

El instrumento de recolección de información (disponible si se solicita a los autores) contiene información sociodemográfica (edad, sexo, estrato socioeconómico y grado de escolaridad); antecedentes médicos personales (enfermedades sistémicas); hábitos de higiene bucal y de las prótesis dentales (frecuencia de cepillado, utilización de enjuagues, limpieza de la mucosa, remoción nocturna de la prótesis); signos y síntomas al examen clínico de articulación temporomandibular; estado actual de las prótesis (porosidades, pigmentaciones, cálculos, fracturas de la base, dientes caídos, desgaste dentario, textura, extensión de los flancos, retención, estabilidad y soporte); revisiones después de la adaptación de la prótesis; frecuencia de visita al odontólogo y tiempo de uso.

Se consideró también como variable la calidad de vida relacionada con la salud bucal (CVRSB), por

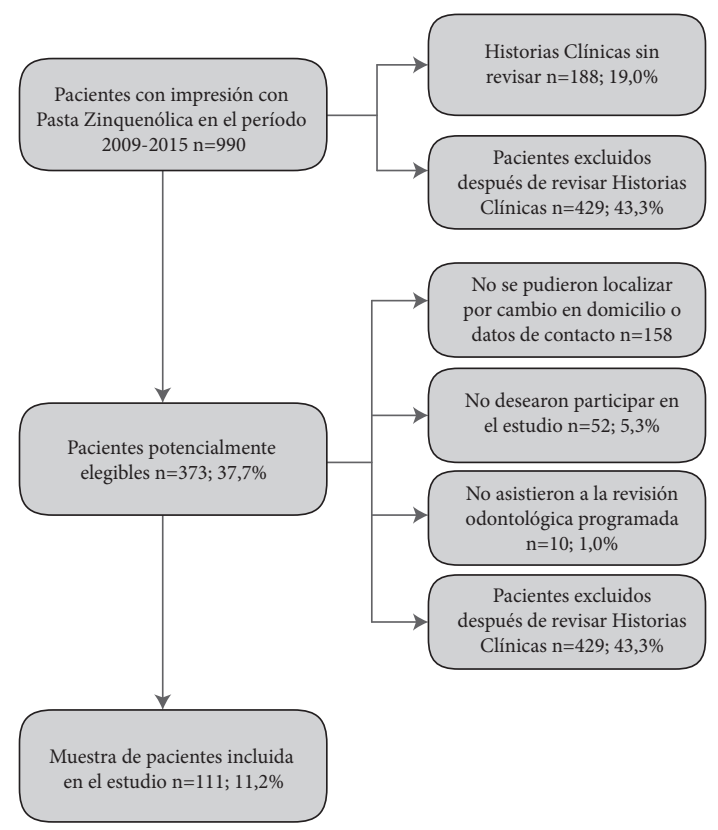

Figura 1. Proceso de selección de los pacientes incluidos en el estudio (2009- 2015)

Fuente: elaboración propia medio del Índice de Salud Oral Geriátrico (GOHAI) [24]. Este es un instrumento que consta de 12 preguntas con varias opciones de respuesta; se establece un puntaje que va en una escala de 0 a 60 (0 corresponde a la peor valoración de CVRSB, y 60 la mejor). Las escalas del GOHAI se dividieron en tres categorías: alta (57 a 60$)$, moderada (51 a 56$)$, y baja $(\leq 50)$.

Se describieron las variables del estudio mediante frecuencias absolutas y relativas, haciendo análisis separados para hombres y mujeres. Para la variable CVRSB, de acuerdo con los puntajes del índice GOHAI, se calcularon medidas de resumen (media, mediana, valores mínimo y máximo), y frecuencias de alta, media y baja CVRsB. Para el análisis de los datos se utilizaron los programas Excel para Windows, sPss 21.0 y EPIDAT 3.1.

Este estudio estuvo acorde con la normativa nacional e internacional para investigación en seres humanos, y según la Resolución 8430 del Ministerio de Protección Social y Salud (antes Ministerio de Salud) se considera una investigación de riesgo mínimo. Todos los pacientes dieron su consentimiento oral y escrito para participar. Los pacientes que requerían cambio de sus prótesis, o algún tipo de tratamiento, fueron remitidos para su atención, y en algunos casos se realizaron medidas de educación en higiene bucal y algún tipo de mantenimiento que podría realizarse durante la cita de revisión odontológica. Este proyecto fue aprobado como ejercicio académico durante el pregrado de Odontología, Medellín, de la Universidad de Antioquia. En la redacción del manuscrito se tuvieron en cuenta las pautas de la guía STROBE para estudios observacionales, así como su adaptación para estudios descriptivos o transversales $[25,26]$.

\section{Resultados}

El $66,7 \%$ de los pacientes evaluados eran hombres, los rangos de edad de la población revisada estuvieron entre los 49 y 91 años, en los que el mayor porcentaje $(38,7 \%)$ se encontró entre los 49 a 64 años, con diferencias muy bajas con los demás rangos. La mayoría de la población evaluada $(98,2 \%)$, vivía en los estratos bajo y medio. En cuanto a la afiliación al Sistema General de Seguridad Social en Salud, el $73,8 \%$ pertenecían al régimen contributivo. El $82,9 \%$ de los pacientes solo cursaron estudios básicos (véase la tabla 1). 
Tabla 1. Características sociodemográficas en los pacientes portadores de prótesis dental total bimaxilar incluidos en el estudio. Medellín, Colombia, 2009-2015 $(\mathrm{n}=111)$

\begin{tabular}{|c|c|c|c|c|c|c|}
\hline \multirow{2}{*}{ Variables } & \multicolumn{2}{|c|}{ Hombres } & \multicolumn{2}{|c|}{ Mujeres } & \multicolumn{2}{|c|}{ Total } \\
\hline & $\mathbf{n}$ & $\%$ & $\mathbf{n}$ & $\%$ & $\mathbf{n}$ & $\%$ \\
\hline \multicolumn{7}{|l|}{ Edad } \\
\hline $49-64$ & 28 & 37,8 & 15 & 40,5 & 43 & 38,7 \\
\hline $65-74$ & 20 & 27,0 & 16 & 43,2 & 36 & 32,4 \\
\hline 75 y más & 26 & 35,1 & 6 & 16,2 & 32 & 28,8 \\
\hline \multicolumn{7}{|c|}{ Afiliación a la seguridad social } \\
\hline Contributivo (cotizante) & 29 & 39,2 & 22 & 59,5 & 51 & 45,9 \\
\hline $\begin{array}{l}\text { Contributivo (benefi- } \\
\text { ciario) }\end{array}$ & 26 & 35,1 & 5 & 13,5 & 31 & 27,9 \\
\hline Subsidiado & 19 & 25,7 & 10 & 27,0 & 29 & 26,1 \\
\hline \multicolumn{7}{|l|}{ Estrato socioeconómico $^{*}$} \\
\hline Bajo (1-2) & 38 & 51,4 & 15 & 42,9 & 53 & 48,6 \\
\hline Medio (3-4) & 35 & 47,3 & 19 & 54,3 & 54 & 49,5 \\
\hline Alto (5-6) & 1 & 1,4 & 1 & 2,9 & 2 & 1,8 \\
\hline \multicolumn{7}{|l|}{ Grado de escolaridad } \\
\hline Sin estudios & 7 & 9,5 & 4 & 10,8 & 11 & 9,9 \\
\hline Primaria & 52 & 70,3 & 15 & 40,5 & 67 & 60,4 \\
\hline Secundaria & 13 & 17,6 & 12 & 32,4 & 25 & 22,5 \\
\hline Universidad & 2 & 2,7 & 6 & 16,2 & 8 & 7,2 \\
\hline Total & 74 & 66,7 & 37 & 33,3 & 111 & 100,0 \\
\hline
\end{tabular}

Fuente: elaboración propia

Se encontró que en un $42,3 \%$ relataban ser exfumadores (fumadores 18\%), y el 40\% afirmó no haber consumido licor. Más de la mitad de la población $(69,4 \%)$ reportó tener un estado de salud bueno, sin embargo, se encontró que el 53,2\% relató sufrir de hipertensión, el 23,4\% de diabetes, el 12,6\% de artritis reumatoide, y el $13,5 \%$ de problemas tiroideos (véase la tabla 2).

En cuanto a las condiciones de higiene bucal (figura 1), tres cuartas partes del total de pacientes evaluados manifestaron limpiarse la mucosa alveolar, tanto superior, como inferior, siendo las mujeres las que más practican este hábito $(83,8 \%)$, y menos de un cuarto de la población reportó el uso de enjuague bucal como complemento de higiene. Tanto los hombres como las mujeres (77,5\%) utilizan crema dental para la higiene de la prótesis, y aproximadamente la mitad de la población de estudio se remueve las prótesis en la noche, siendo mayor el porcentaje de remoción de la prótesis inferior $(56,8 \%)$.
Tabla 2. Antecedentes médicos personales en los pacientes portadores de prótesis dental total bimaxilar incluidos en el estudio. Medellín, Colombia, 2009-2015 $(\mathrm{n}=111)$

\begin{tabular}{|c|c|c|c|c|c|c|}
\hline \multirow{2}{*}{ Variables } & \multicolumn{2}{|c|}{ Hombres } & \multicolumn{2}{|c|}{ Mujeres } & \multicolumn{2}{|c|}{ Total } \\
\hline & $\mathbf{n}$ & $\%$ & $\mathbf{n}$ & $\%$ & $\mathbf{n}$ & $\%$ \\
\hline \multicolumn{7}{|l|}{ Consumo de cigarrillo } \\
\hline Fumador & 12 & 16,2 & 8 & 21,6 & 20 & 18,0 \\
\hline No fumador & 34 & 45,9 & 7 & 18,9 & 41 & 36,9 \\
\hline Fumador ocasional & 2 & 2,7 & 1 & 2,7 & 3 & 2,7 \\
\hline Exfumador & 26 & 35,1 & 21 & 56,8 & 47 & 42,3 \\
\hline \multicolumn{7}{|l|}{ Consumo de alcohol $^{*}$} \\
\hline Bebedor & 1 & 1,4 & 4 & 11,1 & 5 & 4,5 \\
\hline No bebedor & 37 & 50,0 & 7 & 19,4 & 44 & 40,0 \\
\hline Bebedor ocasional & 19 & 25,7 & 11 & 30,6 & 30 & 27,3 \\
\hline Exbebedor & 17 & 23,0 & 14 & 38,9 & 31 & 28,2 \\
\hline \multicolumn{7}{|c|}{ Condición de salud actual } \\
\hline Bueno & 49 & 66,2 & 28 & 75,7 & 77 & 69,4 \\
\hline Regular & 23 & 31,1 & 9 & 24,3 & 32 & 28,8 \\
\hline Malo & 2 & 2,7 & 0 & 0,0 & 2 & 1,8 \\
\hline \multicolumn{7}{|l|}{ Condiciones sistémicas } \\
\hline Hipertensión & 41 & 55,4 & 18 & 48,6 & 59 & 53,2 \\
\hline Diabetes & 18 & 24,3 & 8 & 21,6 & 26 & 23,4 \\
\hline Artritis reumatoide & 11 & 14,9 & 3 & 8,1 & 14 & 12,6 \\
\hline Problemas en tiroides & 11 & 14,9 & 4 & 10,8 & 15 & 13,5 \\
\hline
\end{tabular}

Fuente: elaboración propia

Con relación al examen clínico de articulación temporomandibular (figura 2), casi la mitad de la población estudiada presentaba ruido articular (45,9\%), y el músculo al que refirieron más dolor durante la palpación fue el pterigoideo externo $(28,8 \%)$, siendo el porcentaje más alto en hombres que en mujeres.

Dentro de las características protésicas (véase la tabla 3), se encontraron con mayor frecuencia y en orden decreciente pigmentaciones, cálculos y porosidades. La textura de ambas prótesis fue lisa para alrededor de tres cuartas partes de la población. La extensión de los flancos en las prótesis superiores fue normal para un 54,5\%, y sobreextendidos en un $50,1 \%$ para las prótesis inferiores. Las fracturas de base y los dientes caídos se presentaron en bajos porcentajes para ambas prótesis, mientras que un poco más de la mitad presentaron desgaste dentario. En cuanto a las características de retención, 
estabilidad y soporte de la prótesis superior, se halló que el $91,0 \%$, el $89,2 \%$ y el $84,7 \%$, respectivamente, se encontraban adecuadas. En contrapeso, la retención y estabilidad de la prótesis inferior se mostró escasa $(74,8 \%)$, e inadecuada $(68,5 \%)$, mientras que el soporte se halló bueno en un 71,2\%. La dimensión vertical se constató neutra en un poco más de la mitad de la población estudiada $(51,4 \%)$.
Con respecto a las características relacionadas con el uso de las prótesis (véase la tabla 4), se encuentran porcentajes similares entre el tiempo de uso de la prótesis superior y de la inferior, con el mayor porcentaje (45\%) ubicado en el rango de 25 a 48 meses. Una buena parte de la población encuestada (57,7\%) aduce asistir a revisiones odontológicas cada tres años o más, mientras que solo una

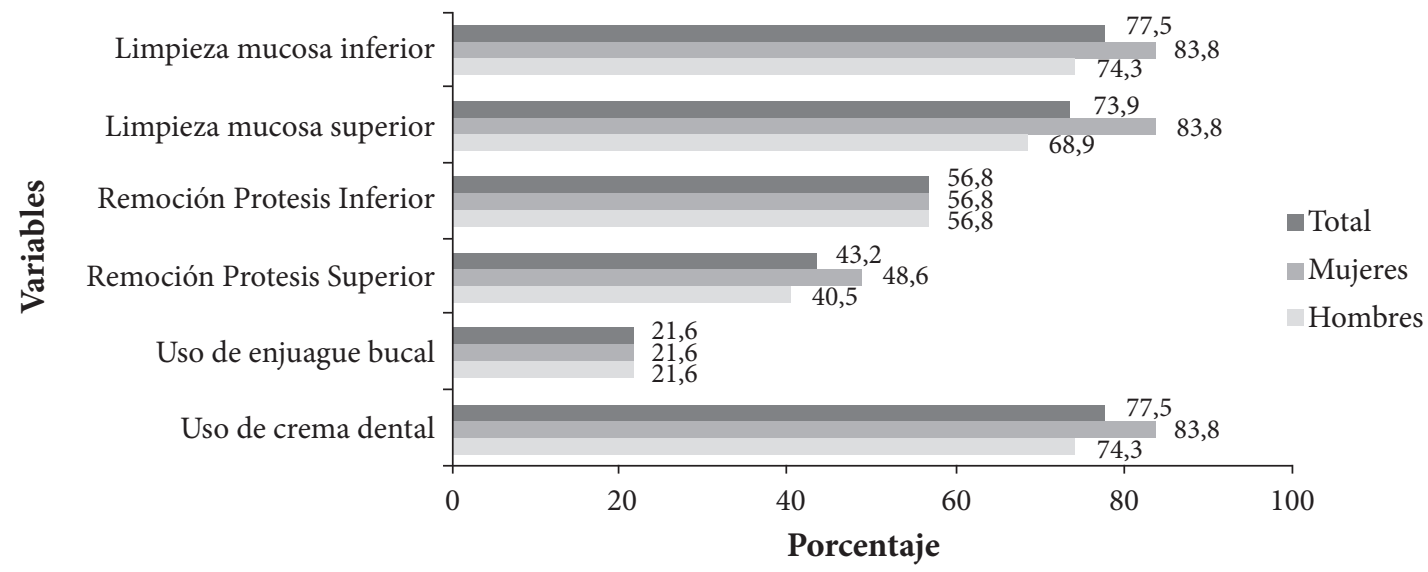

Figura 1. Condiciones de higiene bucal en los pacientes portadores de prótesis dental total bimaxilar incluidos en el estudio. Medellín, Colombia, 2009- 2015 (n=111)

Fuente: elaboración propia

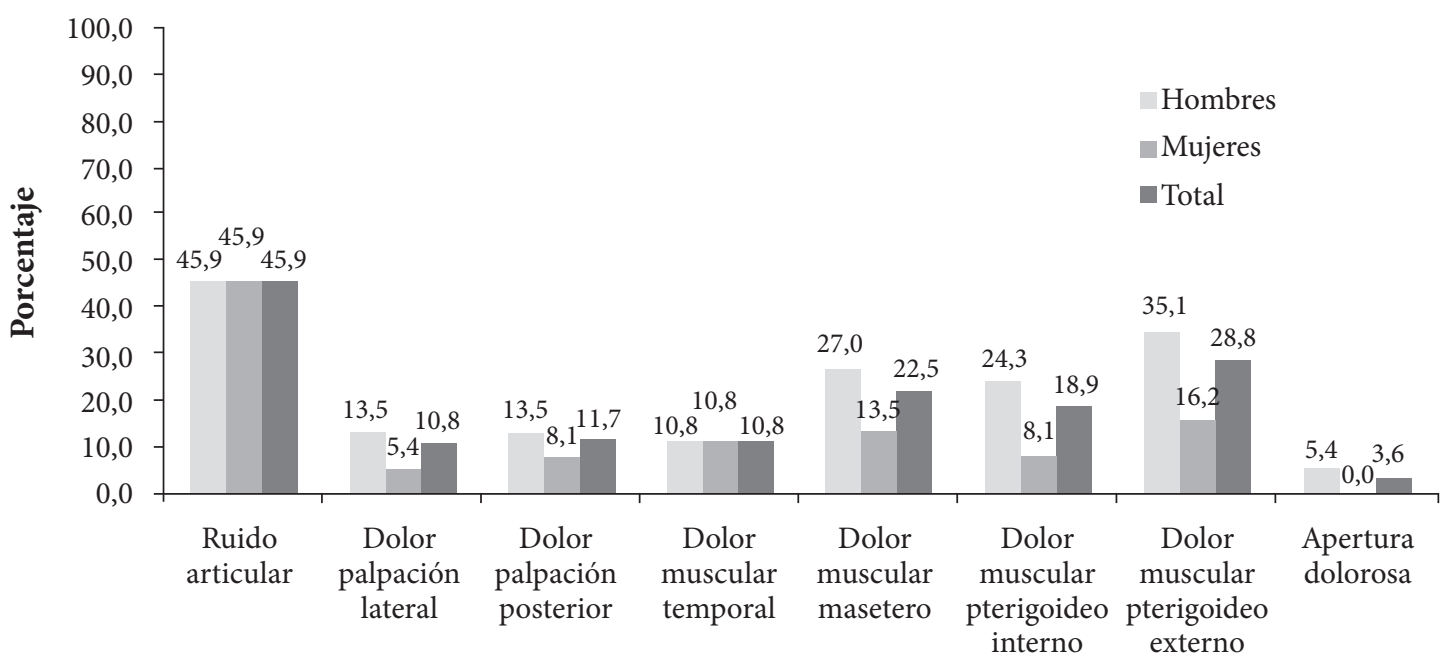

Signos y síntomas al examen clínico

Figura 2. Signos y síntomas de problemas de la articulación temporomandibular según examen clínico en los pacientes portadores de prótesis dental total bimaxilar incluidos en el estudio. Medellín, Colombia, 2009- 2015 (n=111)

Fuente: elaboración propia 
minoría acude a revisión cada tres o seis meses. La población encuestada expresó mayor satisfacción con la prótesis superior comparada con la inferior $(89,2 \%$ respecto a $61,3 \%)$. Además, las mujeres se mostraron más satisfechas que los hombres en relación con sus prótesis (véase la tabla 4).

Tabla 3. Condiciones de las prótesis dentales totales bimaxilares en los pacientes incluidos en el estudio. Medellín, Colombia, 2009-2015 ( $\mathrm{n}=111)$

\begin{tabular}{|c|c|c|c|c|c|c|}
\hline \multirow{2}{*}{ Variables } & \multicolumn{2}{|c|}{ Hombres } & \multicolumn{2}{|c|}{ Mujeres } & \multicolumn{2}{|c|}{ Total } \\
\hline & $\mathbf{n}$ & $\%$ & $\mathbf{n}$ & $\%$ & $\mathrm{n}$ & $\%$ \\
\hline \multicolumn{7}{|c|}{ Prótesis total superior } \\
\hline Porosidades & 26 & 35,1 & 17 & 45,9 & 43 & 38,7 \\
\hline Pigmentaciones & 39 & 52,7 & 23 & 62,2 & 62 & 55,9 \\
\hline Cálculos & 34 & 45,9 & 20 & 54,1 & 54 & 48,6 \\
\hline Fracturas de base & 10 & 13,5 & 5 & 13,5 & 15 & 13,5 \\
\hline Dientes caídos & 4 & 5,4 & 0 & 0,0 & 4 & 3,6 \\
\hline Desgaste dentario & 37 & 50,0 & 21 & 56,8 & 58 & 52,3 \\
\hline \multicolumn{7}{|l|}{ Textura } \\
\hline Lisa & 65 & 87,8 & 32 & 86,5 & 97 & 87,4 \\
\hline Rugosa & 9 & 12,2 & 5 & 13,5 & 14 & 12,6 \\
\hline \multicolumn{7}{|l|}{ Extensión flancos } \\
\hline Normal & 40 & 54,1 & 20 & 54,1 & 60 & 54,1 \\
\hline Sobreextendidos & 22 & 29,7 & 13 & 35,1 & 35 & 31,5 \\
\hline Subextendidos & 12 & 16,2 & 4 & 10,8 & 16 & 14,4 \\
\hline \multicolumn{7}{|l|}{ Retención } \\
\hline Buena & 68 & 91,9 & 33 & 89,2 & 101 & 91,0 \\
\hline Escasa & 6 & 8,1 & 4 & 10,8 & 10 & 9,0 \\
\hline \multicolumn{7}{|l|}{ Estabilidad } \\
\hline Adecuada & 68 & 91,9 & 31 & 83,8 & 99 & 89,2 \\
\hline Inadecuada & 6 & 8,1 & 6 & 16,2 & 12 & 10,8 \\
\hline \multicolumn{7}{|l|}{ Soporte } \\
\hline Bueno & 66 & 89,2 & 28 & 75,7 & 94 & 84,7 \\
\hline Escaso & 8 & 10,8 & 9 & 24,3 & 17 & 15,3 \\
\hline \multicolumn{7}{|c|}{ Prótesis total inferior } \\
\hline Porosidades & 38 & 51,4 & 14 & 37,8 & 52 & 46,8 \\
\hline Pigmentaciones & 39 & 52,7 & 18 & 47,3 & 57 & 51,4 \\
\hline Cálculos & 34 & 45,9 & 22 & 59,5 & 56 & 50,5 \\
\hline Fracturas de base & 6 & 8,1 & 4 & 10,8 & 10 & 9,0 \\
\hline Dientes caídos & 3 & 4,1 & 1 & 2,7 & 4 & 3,6 \\
\hline Desgaste dentario & 39 & 52,7 & 19 & 51,4 & 58 & 52,3 \\
\hline \multicolumn{7}{|l|}{ Textura } \\
\hline Lisa & 55 & 74,3 & 31 & 83,8 & 86 & 77,5 \\
\hline Rugosa & 19 & 25,7 & 6 & 16,2 & 25 & 22,5 \\
\hline
\end{tabular}

\begin{tabular}{|c|c|c|c|c|c|c|}
\hline \multirow{2}{*}{ Variables } & \multicolumn{2}{|c|}{ Hombres } & \multicolumn{2}{|c|}{ Mujeres } & \multicolumn{2}{|c|}{ Total } \\
\hline & $\mathrm{n}$ & $\%$ & n & $\%$ & $\mathrm{n}$ & $\%$ \\
\hline \multicolumn{7}{|l|}{ Extensión flancos } \\
\hline Normal & 30 & 40,5 & 21 & 56,8 & 51 & 45,9 \\
\hline Sobreextendidos & 42 & 56,8 & 14 & 37,8 & 56 & 50,5 \\
\hline Subextendidos & 2 & 2,7 & 2 & 5,4 & 4 & 3,6 \\
\hline \multicolumn{7}{|l|}{ Retención } \\
\hline Buena & 17 & 23,0 & 11 & 29,7 & 28 & 25,2 \\
\hline Escasa & 57 & 77,0 & 26 & 70,3 & 83 & 74,8 \\
\hline \multicolumn{7}{|l|}{ Estabilidad } \\
\hline Adecuada & 24 & 32,4 & 11 & 29,7 & 35 & 31,5 \\
\hline Inadecuada & 50 & 67,6 & 26 & 70,3 & 76 & 68,5 \\
\hline \multicolumn{7}{|l|}{ Soporte } \\
\hline Bueno & 52 & 70,3 & 27 & 73,0 & 79 & 71,2 \\
\hline Escaso & 22 & 29,7 & 10 & 27,0 & 32 & 28,8 \\
\hline \multicolumn{7}{|l|}{ Dimensión vertical } \\
\hline Aumentada & 22 & 29,7 & 17 & 45,9 & 39 & 35,1 \\
\hline Neutra & 38 & 51,4 & 19 & 51,4 & 57 & 51,4 \\
\hline Disminuida & 14 & 18,9 & 14 & 2,7 & 15 & 13,5 \\
\hline
\end{tabular}

Fuente: elaboración propia

Tabla 4. Uso y satisfacción de las prótesis dentales totales bimaxilares en los pacientes incluidos en el estudio.

Medellín, Colombia, 2009-2015 ( $\mathrm{n=111)}$

\begin{tabular}{|c|c|c|c|c|c|c|}
\hline \multirow{2}{*}{ Variables } & \multicolumn{2}{|c|}{ Hombres } & \multicolumn{2}{|c|}{ Mujeres } & \multicolumn{2}{|c|}{ Total } \\
\hline & $\mathrm{n}$ & $\%$ & $\mathrm{n}$ & $\%$ & n & $\%$ \\
\hline \multicolumn{7}{|c|}{ Tiempo de uso de prótesis superior (meses) } \\
\hline$\leq 24$ & 19 & 25,7 & 15 & 40,5 & 34 & 30,6 \\
\hline 25- 48 & 35 & 47,3 & 15 & 40,5 & 50 & 45 \\
\hline$\geq 49$ & 20 & 27,0 & 7 & 18,9 & 27 & 24,3 \\
\hline \multicolumn{7}{|c|}{ Tiempo de uso de prótesis inferior (meses) } \\
\hline$\leq 24$ & 20 & 27,0 & 15 & 40,5 & 35 & 31,5 \\
\hline 25- 48 & 35 & 47,3 & 15 & 40,5 & 50 & 45,0 \\
\hline$\geq 49$ & 19 & 25,7 & 7 & 18,9 & 26 & 23,4 \\
\hline \multicolumn{7}{|c|}{ Tiempo de la última consulta (meses) } \\
\hline$\leq 12$ & 44 & 59,5 & 21 & 56,8 & 65 & 58,6 \\
\hline $13-24$ & 12 & 16,2 & 9 & 24,3 & 21 & 18,9 \\
\hline$\geq 25$ & 18 & 24,3 & 7 & 18,9 & 25 & 22,5 \\
\hline \multicolumn{7}{|c|}{ Revisión de prótesis totales } \\
\hline Cada tres meses & 1 & 1,4 & 0 & 0,0 & 1 & 0,9 \\
\hline Cada seis meses & 2 & 2,7 & 1 & 2,7 & 3 & 2,7 \\
\hline Entre uno y tres años & 23 & 31,1 & 20 & 54,1 & 43 & 38,7 \\
\hline Más de tres años & 48 & 64,9 & 16 & 43,2 & 64 & 57,7 \\
\hline
\end{tabular}


(viene)

\begin{tabular}{|l|c|c|c|c|c|c|c|}
\hline \multirow{2}{*}{ Variables } & Hombres & \multicolumn{2}{c|}{ Mujeres } & \multicolumn{2}{c|}{ Total } \\
\cline { 2 - 8 } & $\mathbf{n}$ & $\%$ & $\mathbf{n}$ & $\%$ & $\mathbf{n}$ & $\%$ \\
\hline Satisfacción prótesis superior \\
\hline Sí & 64 & 86,5 & 35 & 94,6 & 99 & 89,2 \\
\hline No & 10 & 13,5 & 2 & 5,4 & 12 & 10,8 \\
\hline Satisfacción prótesis inferior \\
\hline Sí & 44 & 59,5 & 24 & 64,9 & 68 & 61,3 \\
\hline No & 30 & 40,5 & 13 & 35,1 & 43 & 38,7 \\
\hline
\end{tabular}

Fuente: elaboración propia

En relación con la calidad de vida y con respecto a la salud bucal (medida con el test GOHAI, véanse las tablas 5 y 6 ), se evidencian puntajes iguales o por debajo de 56 (promedio: $44 \pm 7,5$ ). Esto representa más de las tres cuartas partes de la población encuestada (77,5\%), de manera que tienen una baja calidad de vida (un poco mayor en hombres). Sin embargo, en el rango moderado el mayor porcentaje estuvo representado por mujeres. Ninguno de los participantes reportó su calidad de vida relacionada con la salud bucal como buena.

Tabla 5. Promedio (desviación estándar) y mediana (rango intercuartílico) de los puntajes del test GOHAI en los pacientes portadores de prótesis dental total bimaxilar incluidos en el estudio. Medellín, Colombia, 2009- $2015(n=111)^{*}$

\begin{tabular}{|l|c|c|c|c|}
\hline \multirow{2}{*}{ Variables } & \multicolumn{2}{|c|}{ Puntaje GOHAI } & \multicolumn{2}{|c|}{} \\
\cline { 2 - 5 } & $\mathrm{X}(\mathrm{DS})$ & $\mathrm{Me}(\mathrm{Q} 3$ - Q1) & Mín & Máx \\
\hline Hombres & $44(7,6)$ & $46(50-39)$ & 25 & 54 \\
\hline Mujeres & $45(7,6)$ & $47(51-41)$ & 21 & 54 \\
\hline Total & $44(7,5)$ & $47(50-40)$ & 21 & 54 \\
\hline
\end{tabular}

X: Promedio; DS: desviación estándar, Mín: valor mínimo; Máx valor máximo.

Fuente: elaboración propia

Tabla 6. Calidad de vida relacionada con la salud bucal (CVRsB), según los criterios del test GOHAI en los pacientes portadores de prótesis dental total bimaxilar incluidos en el estudio. Medellín, Colombia, 2009-2015 $(\mathrm{n}=111)^{*}$

\begin{tabular}{|l|l|l|l|l|l|l|}
\hline $\begin{array}{c}\text { Calidad de vida rela- } \\
\text { cionada con la salud } \\
\text { bucal según GOHAI }\end{array}$ & \multicolumn{2}{|c|}{ Hombres } & \multicolumn{2}{|c|}{ Mujeres } & \multicolumn{2}{|c|}{ Total } \\
\cline { 2 - 8 } & 15 & 20,3 & 10 & 27,0 & 25 & 22,5 \\
\hline Moderada (51-56) & 59 & 79,7 & 27 & 73,0 & 86 & 77,5 \\
\hline Baja ( $\leq 50)$ &
\end{tabular}

Fuente: elaboración propia

\section{Discusión}

Este estudio analizó las condiciones de salud bucal en los pacientes portadores de prótesis dental bimaxilar atendidos en la Facultad de Odontología de la Universidad de Antioquia, en el período 2009. 2015. Como principales hallazgos, se encontró que la población evaluada presenta ruidos articulares, dolor muscular en la región de la ATM, así como algunas deficiencias en higiene bucal (en algunos casos). En cuanto a las prótesis dentales, se observaron casos de desgaste dentario $y$, con respecto a las cualidades funcionales de las prótesis, se encontraron fallas en la retención, especialmente en la prótesis inferior. Un porcentaje importante de la población asiste con baja frecuencia a revisiones odontológicas, y se encontró que los pacientes estaban satisfechos con sus prótesis, especialmente con la superior. La mayoría de la población encuestada evaluó su calidad de vida relacionada con la salud bucal como baja. Como institución formadora del recurso humano bajo el modelo docencia-servicio, los resultados permiten evaluar la necesidad de los planes de seguimiento a los tratamientos efectuados por los estudiantes.

La identificación del porcentaje de población edentada total en la sociedad colombiana según datos del ENSAB IV, es del 33\% en adultos de 65-79 años, de los cuales el mayor porcentaje de edentados totales está vinculado a otros regímenes (7,7\%). Estos resultados contrastan con lo que se encontró en este estudio respecto a la afiliación al Sistema General de Seguridad Social en Salud (sgsss), en el que la mayor proporción de la población encuestada pertenece al régimen contributivo, pero acuden a realizarse el tratamiento de forma particular a la Facultad de Odontología de la Universidad de Antioquia. Como posibles explicaciones a lo anterior, podrían tenerse en cuenta factores tales como la gran acogida de los tratamientos en la población del área de influencia de la facultad, dada su calidad y economía, así como la falta de cubrimiento de estos en los planes de beneficio del sGsss, en el cual solo se incluye la colocación de prótesis dentales mucosoportadas totales para cotizantes o beneficiarios con un ingreso base de cotización igual o menor a dos salarios mínimos mensuales legales vigentes [27].

El total de la muestra fue de 111 pacientes, con un rango de edad entre los 45 y los 91 años. El estudio de Carmen Perea et al. [28] relaciona los 
factores sociodemográficos al estado de las prótesis dentales bimaxilares, y encontró que el nivel educativo predominante era el constituido por los grados correspondientes a básica primaria, al igual que en los datos encontrados en la presente investigación. Esto podría asociarse al hecho de que en décadas pasadas el acceso a la educación superior - en especial para el género femenino- era escaso, y se ve reflejado en que casi la mitad de la población femenina cursó solo estudios básicos. La educación tiene un impacto en el estado de la salud bucal, en concordancia con los resultados expresados en el ENSAB IV [11], y de otros estudios en población adulta y adulta mayor [22, 29].

Estudios que evalúan hábitos de higiene [19, 30] y salud oral [28] en pacientes portadores de prótesis total bimaxilar, encontraron resultados similares. La mayoría de participantes en estas investigaciones fueron del sexo masculino y con una edad cercana a los 60 años. Referente a las prácticas de autocuidado, se ha demostrado que el género juega un factor importante, mostrando mejor destreza en las mujeres en comparación con los hombres [29].

En la presente investigación se halló que el principal complemento de higiene bucal es la crema dental. Estos resultados se pueden relacionar con los encontrados por Patel et al. [31], en la cual 90 de 106 pacientes señalaron como método más utilizado el cepillo y el agua, junto con la crema dental. Como dato adicional, 55 pacientes adujeron no haber recibido instrucciones de higiene. Otros estudios, como, por ejemplo, el de Saha et al. (30), encontraron que solo el $19,1 \%$ de los sujetos mantuvieron una higiene con agua, cepillo dental y jabón. De estos hallazgos, se insiste en la necesidad de reforzar las medidas de educación en higiene de las prótesis dentales, ya que la limpieza con dentífrico puede generar daños en el material de la prótesis debido a su potencial abrasivo [32, 33].

En cuanto a la remoción de la prótesis durante la noche, los resultados están en concordancia con el estudio piloto realizado previamente en la facultad [20]. Como ejemplo de esto, Takamiya et al. [34] encontraron porcentajes similares, ya que el $55,8 \%$ de la población se removía la prótesis en la noche. Por otro lado, Peracini et al. reportaron un $41,5 \%$ de pacientes que se remueven la prótesis [19], mientras que Evren et al. reportan cifras del 31\% [35]. Estos datos explican las dificultades con el mantenimiento de las prótesis dentales, en tanto se presentan pocas indicaciones suministradas a los pacientes.

Se encontró que menos de un cuarto de la población estaba conformada por fumadores, siendo las mujeres quienes más practican este hábito, en comparación con los hombres. El tabaquismo es uno de los factores ambientales que aumenta la susceptibilidad del individuo al desarrollo de la enfermedad periodontal, y que, de acuerdo con la literatura $[36,38]$, es una de las principales causas de pérdida dental, debido al deterioro causado en los tejidos de soporte dentales. Esto podría justificar en parte la condición actual de los pacientes incluidos en el estudio a nivel retrospectivo, si se tiene en cuenta que una buena parte de los pacientes encuestados relataron ser exfumadores.

En los datos aportados por la investigación con relación al reporte de enfermedades sistémicas, más de la mitad de los pacientes consideran tener un estado de salud bueno, a pesar del alto porcentaje de hipertensión arterial (HTA) encontrado. Estos datos coinciden con un estudio de seguimiento realizado en Estados Unidos [39], en el cual se halló que la prevalencia de HTA entre los 60 y 69 años de edad era del $60 \%$, del $72 \%$ para los encuestados entre 70 y 79 años, y ascendía al 77\% para los mayores de 80. En lo que respecta a la artritis, se encontró que menos de un cuarto de la población la sufría, lo cual no coincide con otras investigaciones en las que se reportan datos cercanos al 50\% [40]. En cuanto a la diabetes, se encontró que un poco menos de un cuarto de los pacientes evaluados presentaron dicha patología, siendo más prevalente en mujeres que en hombres. En un estudio llevado a cabo en México se encontró una prevalencia de diabetes mayor en mujeres que en hombres [41]. Por último, en cuanto a la tiroides, se encontró que menos de un quinto de los pacientes reportaron verse afectados por este padecimiento, lo cual concuerda con lo referido por un estudio realizado en un centro de salud urbano en España [42], el cual reporta una prevalencia del 13\%. Es importante analizar en los pacientes todo lo relacionado con el estado de salud bucal y su correspondencia con enfermedades sistémicas que puedan afectar el desarrollo del tratamiento odontológico implementado.

En lo que se refiere a los signos y síntomas clínicos asociados a disfunciones en la ATM, se encontró mayor porcentaje de alteraciones en hombres, en contraste con lo relatado en estudios realizados en Perú [43] y Cuba [44]. El signo que se encontró 
con mayor frecuencia, tanto en hombres, como en mujeres, fue el ruido articular, dato reportado en investigaciones similares $[43,46]$. La pérdida dental se considera como un factor generador de diversos trastornos temporomandibulares, debido a que la posición mandibular se altera $[47,48]$. Posibles explicaciones radican sobre el hecho de que los pacientes portadores de prótesis dentales bimaxilares tienden a presentar mayor cantidad de signos y síntomas asociados a desórdenes temporomandibulares, en razón a las condiciones propias de la degeneración tisular y a la tendencia a la pérdida dental. Por tanto, se debe tener especial cuidado y control a fin de evitar molestias crónicas y degenerativas.

La dimensión vertical, tanto en pacientes desdentados totales, como parciales, generalmente varía según la rehabilitación realizada. Mohindra y Bulman [49] afirman que la estética facial en estos pacientes mejora considerablemente al aumentar la dimensión vertical. En el presente estudio se encontró que un poco más de la mitad de los pacientes revisados presentaba una dimensión vertical neutra, en contraste con lo relatado por Quiroga del Pozo et al. [50], en cuyo estudio el $60 \%$ de los pacientes presentaban una dimensión vertical aumentada. El aumento de la dimensión vertical se relaciona con el deterioro de la eficacia masticatoria, aceleración de la reabsorción del reborde alveolar y afección en el funcionamiento biomecánico.

Según los datos reportados en un estudio previo [51], el 67,5\% de la población había usado la prótesis por un período de tiempo mayor de seis años. En la facultad, el mayor porcentaje se concentró en el rango de 25-48 meses. Respecto a las revisiones, la población encuestada aduce acudir a revisiones en un período igual o mayor a tres años, datos que se corresponden con lo reportado en un estudio realizado en Estados Unidos [52], en el cual más del $25 \%$ de la población de adultos mayores no acude a consulta odontológica hace más de cinco años, y aquellos que usan dentaduras completas visitan cuatro veces menos al odontólogo con respecto a los que tienen dientes remanentes.

La satisfacción con relación al uso de las prótesis, tanto inferior, como superior, en general fue buena, en especial para la prótesis superior, para la cual se encontró que nueve de cada 10 pacientes relataron estar satisfechos, a pesar de llevar más de 12 meses de uso. La satisfacción no solo está asociada a la calidad de fabricación de la prótesis, sino también a factores psicológicos y emocionales relacionados con su uso y a las condiciones físicas y biológicas locales de cada paciente [53]. Otros estudios sugieren que la satisfacción de las prótesis totales convencionales son un reflejo de la satisfacción con la prótesis inferior $[54,55]$. Estos hallazgos contrastan con la retención y estabilidad inadecuada encontrada en la mayoría de las prótesis inferiores evaluadas.

Por último, se encontraron algunas características poco deseables en las prótesis dentales tales como pigmentaciones, porosidades, cálculos y desgastes. Estos resultados concuerdan con el estudio piloto previo [20]. En consonancia con lo encontrado respecto a la situación de higiene bucal y la estabilidad en las prótesis, se evidencian las dificultades para hacer un control y seguimiento de los pacientes. En esto intervienen características del sistema de atención en el modelo docencia-servicio, teniendo en cuenta la alta demanda de tratamientos de rehabilitación de la facultad, así como factores individuales propios de la cultura y del proceso de envejecimiento, ya que se evidencian problemas sistémicos y pérdida de la destreza manual en los pacientes adultos mayores $[19,20]$.

El test GOHAI permitió evaluar diferentes dimensiones en las actividades cotidianas relacionadas con la salud bucal que afectan la calidad de vida (físicas y psicosociales). La provisión de prótesis dentales en correcto estado [17] aumenta la satisfacción de las personas y, por ende, su calidad de vida. Más de tres cuartas partes de los pacientes incluidos en el estudio reportaron su CVRSB como baja. Muchos pacientes, al no tener sus prótesis en buen estado, relatan inconformidad, dolor, problemas de masticación y problemas sociales. No obstante, se requiere profundizar con mayor detalle en los factores que pueden afectar la CVRSB en estos pacientes, ya que se considera un concepto multidimensional que tiene en cuenta factores individuales y factores del contexto social y económico [56].

Por otra parte, es importante comentar las fortalezas y limitaciones del presente estudio. Un aspecto a considerar tiene que ver con la naturaleza del diseño y el diseño muestral. Al ser un estudio descriptivo con una muestra por conveniencia, no se puede interpretar causalidad en los resultados, ni inferir en población general, aunque por las características de los participantes los hallazgos se asemejan a otros grupos similares sociodemográficamente. Por otro lado, cabe comentar que 
durante el trabajo de campo se presentaron dificultades para contactar a los pacientes, ya que los teléfonos reportados estaban desactualizados, y se presentaron algunas inconsistencias en la información reportada en las historias clínicas (se notó el poco interés de algunos posibles participantes en acudir a las revisiones). Como fortalezas se destacan la participación de docentes expertos en el área de prótesis total y epidemiología - lo cual permitió un abordaje íntegro-, así como que los instrumentos de recolección de información fueron estandarizados. Superando las limitaciones, este estudio permite el reconocimiento de las necesidades principales en la población objeto de estudio, y propone estrategias acordes con los objetivos de formación del recurso humano en salud bucal, sin detrimento en la calidad de los programas de atención y rehabilitación para este grupo poblacional.

\section{Conclusiones y recomendaciones}

Los pacientes incluidos en el presente estudio presentan alta frecuencia de enfermedades sistémicas. Entre las principales se destacan la hipertensión, la diabetes y los problemas osteoarticulares, una historia de hábitos nocivos tales como el tabaquismo y el consumo de alcohol, y aspectos biológicos asociados al deterioro propio de la edad, lo cual no se refleja en la autopercepción de estos pacientes en relación con su estado de salud general. No obstante, las deficiencias en la higiene bucal y el estado protésico podrían estar asociados a una percepción de mala calidad de vida relacionada con la salud bucal (CVRSB) por parte de estos pacientes.

La situación de salud bucal encontrada, teniendo en cuenta la frecuencia de problemas de ATM, el estado de las prótesis y la percepción de su CVRSB, es reflejo de las condiciones de vulnerabilidad social a la cual se encuentra sometida la población, así como a dificultades en las estrategias de mantenimiento protésico, de educación y motivación, en concordancia con sus características como población adulta mayor.

Los resultados planteados evidencian la necesidad de establecer mecanismos y actividades que permitan mejorar la calidad en la atención en las instituciones formadoras de recurso humano en salud que trabajan bajo el modelo docencia-servicio, y fortalecer los sistemas de vigilancia epidemiológica en la institución, de manera que permitan conocer las condiciones reales de esta población y plantear estrategias acordes con las necesidades encontradas en estos pacientes.

\section{Agradecimientos}

A la Facultad de Odontología de la Universidad de Antioquia, por facilitar la logística para la realización del trabajo de campo, y a los pacientes por su disposición a participar en los exámenes clínicos.

\section{Referencias}

[1] Gerritsen AE, Allen PF, Witter DJ, Bronkhorst EM, Creugers NH. Tooth loss and oral health-related quality of life: a systematic review and meta-analysis. Health Qual Life Outcomes. 2010;8:126.

[2] Kassebaum NJ, Bernabe E, Dahiya M, Bhandari B, Murray CJ, Marcenes W. Global Burden of Severe Tooth Loss: A Systematic Review and Meta-analysis. J Dent Res. 2014;93(7 Suppl):20s-8s.

[3] Minaya-Sánchez M, Medina-Solís CE, Casanova-Rosado JF, Casanova-Rosado AJ, Márquez-Corona $\mathrm{M}$, Islas-Granillo $\mathrm{H}$, et al. Pérdida de dientes y variables del estado periodontal asociadas en hombres policías adultos. Gac Med Mex. 2010;146:264-8.

[4] Arteaga O, Urzúa I, Espinoza I, Muñoz A, Mendoza C. Prevalencia de caries y pérdida de dientes en población de 65 a 74 años de Santiago, Chile. Rev Clin Periodoncia Implantol Rehábil Oral. 2009;2(3): 161-6.

[5] Petersen PE. The World Oral Health Report 2003: continuous improvement of oral health in the 21st century-the approach of the wHo Global Oral Health Programme. Community Dent Oral Epidemiol. 2003;31 Suppl 1:3-23.

[6] Petersen PE, Bourgeois D, Ogawa H, Estupinan-Day $S$, Ndiaye $C$. The global burden of oral diseases and risks to oral health. Bull World Health Organ. 2005;83(9):661-9.

[7] Ribeiro CG, Cascaes AM, Silva AE, Seerig LM, Nascimento GG, Demarco FF. Edentulism, Severe Tooth Loss and Lack of Functional Dentition in Elders: A Study in Southern Brazil. Braz Dent J. 2016;27(3):345-52.

[8] Malheiros AS, Carvalhal ST, Pereira TL, Filho EM, Tonetto MR, Goncalves LM, et al. Association between Tooth Loss and Degree of Temporomandibular Disorders: A Comparative Study. J Contemp Dent Pract. 2016;17(3):235-9. 
[9] Musacchio E, Perissinotto E, Binotto P, Sartori L, Silva-Netto F, Zambon S, et al. Tooth loss in the elderly and its association with nutritional status, socio-economic and lifestyle factors. Acta Odontol Scand. 2007;65(2):78-86.

[10] Peres MA, Tsakos G, Barbato PR, Silva DA, Peres KG. Tooth loss is associated with increased blood pressure in adults-a multidisciplinary population-based study. J Clin Periodontol. 2012;39(9):824-33.

[11] Colombia. Ministerio de Salud y Protección Social. Iv Estudio Nacional de Salud Bucal. ENSAB Iv: Para saber cómo estamos y saber qué hacemos. Bogotá: Ministerio de Salud y Protección Social 2013-2014.

[12] Peres MA, Barbato PR, Reis SC, Freitas CH, Antunes JL. Tooth loss in Brazil: analysis of the 2010 Brazilian Oral Health Survey. Rev Saude Publica. 2013;47 Suppl 3:78-89.

[13] Emami E, de Souza RF, Kabawat M, Feine JS. The impact of edentulism on oral and general health. Int J Dent. 2013;2013:498305.

[14] Luna E, Aristizabal C, Arango AC, Betancur PA, Mejía PN, Ramirez OC. Factores sociales, culturales e históricos que influyeron en la pérdida dental de adultos mayores. Valle de Aburrá 2000. Rev CES Odont. 2009;14(1):13-8.

[15] Lawton B, Rose S, Kieser J, Broadbent J, Sussex P, Thomson M, et al. Disparities in edentulism and tooth loss between Maori and non-Maori New Zealand women. Aust N Z J Public Health. 2008;32(3): 254-60.

[16] Budtz-Jørgensen E. Prosthodontics for the elderly: diagnosis and treatment. Chicago: Quintessence Publishing Co; 1999.

[17] Thalji G, McGraw K, Cooper LF. Maxillary Complete Denture Outcomes: A Systematic Review of Patient-Based Outcomes. Int J Oral Maxillofac Implants. 2016;31 Suppl:s169-81.

[18] Osmari D, Fraga S, Braun KO, Unfer B. Behaviour of the Elderly with Regard to Hygiene Procedures for and Maintenance of Removable Dentures. Oral Health Prev Dent. 2016;14(1):21-6.

[19] Peracini A, Andrade IMd, Paranhos HdFO, Silva CHLd, Souza RFd. Behaviors and hygiene habits of complete denture wearers. Braz Dent J. 2010;21(3):247-52.

[20] Moreno JA, Montoya AF, Gómez DP, Arboleda A, Zea FJ, Agudelo AA. Situación de salud bucal y estado protésico del paciente edentado bimaxilar que acude a la Facultad de Odontología de la Universidad de Antioquia: un estudio piloto. Rev Fac Odontol Univ Antioq. 2012;24(1):22-36.

[21] Ayaz EA, Altintas SH, Turgut S. Effects of cigarette smoke and denture cleaners on the surface rough- ness and color stability of different denture teeth. J Prosthet Dent. 2014;112(2):241-8.

[22] Posada-López A, Agudelo-Suárez AA, Meneses-Gómez EJ. Condiciones de Salud Oral y Estado Protésico de la Población Adulta Mayor Atendida en la Red Hospitalaria Pública de Medellín (Colombia). Int J Odontostomat. 2016;10(1):161-71.

[23] Allen PF. Assessment of oral health related quality of life. Health Qual Life Outcomes. 2003;1:40.

[24] Pinzón-Pulido S, Gil-Montoya J. Validación del Índice de Valoración de Salud Oral en Geriatría en una población geriátrica institucionalizada de Granada. Rev Esp Geriatr y Gerontol. 1999;34(5):273-82.

[25] Von Elm E, Altman DG, Egger M, Pocock SJ, Gøtzsche PC, Vandenbroucke JP. Declaración de la Iniciativa STRoBE (Strengthening the Reporting of Observational studies in Epidemiology): directrices para la comunicación de estudios observacionales. Gac Sanit. 2008;22(2):144-50.

[26] Berra S, Elorza-Ricart JM, Estrada M-D, Sánchez E. Instrumento para la lectura crítica y la evaluación de estudios epidemiológicos transversales. Gac Sanit. 2008;22(5):492-7.

[27] Ministerio de Salud y Protección Social. Plan Obligatorio de Salud (POS) Bogotá: Ministerio de Salud y Protección Social 2016. Disponible en: https://www minsalud.gov.co/salud/ POS/mi-plan/Paginas/MiPlan.aspx

[28] Perea C, Suarez-Garcia MJ, Del Río J, Torres-Lagares D, Montero J, Castillo-Oyague R. Oral health-related quality of life in complete denture wearers depending on their socio-demographic background, prosthetic-related factors and clinical condition. Med Oral Patol Oral Cir Bucal. 2013;18(3):e371-80.

[29] Agudelo-Suárez AA, López-Vergel F, Alzate-Urrea S, López-Orozco C, Espinosa-Herrera E, Posada-López A, et al. Salud bucal y género en relación a la población adulta mayor atendida en la red hospitalaria pública de Medellín: Los puntos de vista del personal de salud. Rev Univ salud. 2016;18(1):58-68

[30] Saha A, Dutta S, Varghese RK, Kharsan V, Agrawal A. A survey assessing modes of maintaining denture hygiene among elderly patients. J Int Soc Prev Community Dent. 2014;4(3):145.

[31] Patel IB, Madan G, Patel B, Solanki K, Chavda R. Behaviours and Hygiene Habits of a sample population of complete denture wearers in Ahmedabad. J Int Oral Health. 2012;4(2):29.

[32] Jagger DC, Harrison A. Denture cleansing--the best approach. Br Dent J. 1995;178(11):413-7.

[33] Dikbas I, Koksal T, Calikkocaoglu S. Investigation of the cleanliness of dentures in a university hospital. Int J Prosthodont. 2006;19(3):294-8. 
[34] Takamiya AS, Monteiro DR, Barao VA, Pero AC, Compagnoni MA, Barbosa DB. Complete denture hygiene and nocturnal wearing habits among patients attending the Prosthodontic Department in a Dental University in Brazil. Gerodontology. 2011;28(2):91-6.

[35] Evren BA, Uludamar A, Ișeri U, Ozkan YK. The association between socioeconomic status, oral hygiene practice, denture stomatitis and oral status in elderly people living different residential homes. Arch Gerontol Geriatr. 2011;53(3):252-7.

[36] Lordelo M. El tabaco y su influencia en el periodonto. Av Periodon Implantol. 2005;17(1):17-24.

[37] Rojas JP, Rojas L, Hidalgo R. Tabaquismo y su efecto en los tejidos periodontales. Rev Clin Periodoncia Implantol Rehábil Oral. 2014;7(2):108-13.

[38] Johnson GK, Slach NA. Impact of tobacco use on periodontal status. J Dent Educ. 2001;65(4):313-21.

[39] Ostchega Y, Dillon CF, Hughes JP, Carroll M, Yoon S. Trends in hypertension prevalence, awareness, treatment, and control in older U.s. adults: data from the National Health and Nutrition Examination Survey 1988 to 2004. J Am Geriatr Soc. 2007;55(7):1056-65.

[40] Berenguer Gouarnalusses M. La salud bucodental en la tercera edad. Medisan. 1999;3(4):53-6.

[41] Shamah-Levy T, Cuevas-Nasu L, Mundo-Rosas V, Morales-Ruán C, Cervantes-Turrubiates L, Villalpando-Hernández S. Estado de salud y nutrición de los adultos mayores en México: resultados de una encuesta probabilística nacional. Salud Publica Mex. 2008;50(5):383-9.

[42] Palacios MS, Vernet MV, López SP, Colomés MF, Blanc MR, Gabriel LP. Enfermedad funcional tiroidea en la población de edad avanzada. Aten Primaria. 2004;34(4):192-7.

[43] Ramírez J, Luz B. Ruidos articulares en pacientes edentulos completos atendidos en la Clínica Estomatológica de la Universidad Nacional de Trujillo, Moche, de agosto a noviembre del 2010. Trujillo (Perú): Universidad de Trujillo; 2011.

[44] Rogelio CG, Ileana GL, Maritza SR. Frecuencia de trastornos temporomandibulares en el área del Policlínico Rampa, Plaza de la Revolución. Rev haban cienc méd. 2009;8(4): Disponible en: http:// scielo.sld.cu/scielo.php?script=sci_arttext\&pi$\mathrm{d}=$ S1729-519X2009000400011\&lng=es\&tlng=es.

[45] Alarco L. Desórdenes témporomandibulares según índice de Fricton y su relación con sintomatología clínica y posición condilar en la Población del dis- trito de Moche, 2007. [Tesis de doctorado en Estomatología]. Trujillo (Perú): Universidad Nacional de Trujillo; 2008.

[46] Rodrigues ET, Suazo IC, Guimaraes AS. Temporomandibular joint sounds and disc dislocations incidence after orotracheal intubation. Clin Cosmet Investig Dent. 2009;1:71-3.

[47] Gutiérrez D. Dolor en la Articulación Temporomandibular en Pacientes Edéntulos Completos que Asistieron a la Clínica Estomatológica de la Universidad Nacional de Trujillo, 2005. Trujillo (Perú): Universidad Nacional de Trujillo; 2006.

[48] Fricton J. Avances recientes en disfunción temporomandibular y dolor orofacial. JADA (Compendio). 1992;1993(3):40-50.

[49] Mohindra NK, Bulman JS. The effect of increasing vertical dimension of occlusion on facial aesthetics. Br Dent J. 2002;192(3):164-8.

[50] Quiroga Del Pozo R, Riquelme Belmar R, Sierra Fuentes M, Del Pozo Bassi J, Quiroga Aravena R. Determinación de la Dimensión Vertical Oclusal en desdentados totales: comparación de métodos convencionales con el craneómetro de Knebelman. Rev Clin Periodoncia Implantol Rehábil Oral. 2012;5(1):20-4.

[51] Torres Curi EM, Miguel Rosales J. Relación entre el uso nocturno y la adaptación a la prótesis estomatológica. Rev Cubana Estomatol. 2000;37(2):77-83.

[52] Ship J. Chapter 42. Oral Cavity. En: Halter J, Ouslander J, Tinetti M, Studenski S, High K, Asthana S (eds.). Hazzard's Geriatric Medicine and Gerontology. $6^{\text {a }}$ ed. New York: McGraw-Hill Prof Med/Tech; 2009.

[53] Carlsson GE. Clinical morbidity and sequelae of treatment with complete dentures. J Prosthet Dent. 1998;79(1):17-23.

[54] Berg E. The influence of some anamnestic, demographic, and clinical variables on patient acceptance of new complete dentures. Acta Odontol Scand. 1984;42(2):119-27.

[55] Berg E. A 2-year follow-up study of patient satisfaction with new complete dentures. J Dent. 1988;16(4):160-5.

[56] Alzate-Urrea S, Agudelo-Suárez AA, López- Vergel F, López-Orozco C, Espinosa-Herrera É, Posada-López A, et al. Calidad de vida y salud bucal: Perspectiva de adultos mayores atendidos en la red hospitalaria pública de Medellín, Colombia. Rev Gerenc Polít Salud. 2015;14(29):83-96. 